\title{
Correlation of trans-Lycopene Measurements by the HPLC Method with the Optothermal and Photoacoustic Signals and the Color Readings of Fresh Tomato Homogenates
}

\author{
Dane Bicanic • Darko Dimitrovski • Svjetlana Luterotti • Ksenija Marković • \\ Charlotte van Twisk • Josephus G. Buijnsters • Otto Dóka
}

Received: 30 July 2009 / Accepted: 13 October 2009 /Published online: 21 November 2009

(C) The Author(s) 2009. This article is published with open access at Springerlink.com

\begin{abstract}
The trans-lycopene content of fresh tomato homogenates was assessed by means of the laser photoacoustic spectroscopy, the laser optothermal window, micro-Raman spectroscopy, and colorimetry; none of these methods require the extraction from the product matrix prior to the analysis. The wet chemistry method (highperformance liquid chromatography) was used as the absolute quantitative method. Analytical figures of merit for all methods were compared statistically; best linear correlation was achieved for the chromaticity index $a^{*}$ and chroma $C^{*}$.
\end{abstract}

Keywords Fresh tomato trans-lycopene content .

Photoacoustic spectroscopy · Optothermal window · Color measurements

D. Bicanic $(\bowtie)$

Laboratory of Biophysics, Wageningen University,

Dreijenlaan 3-Transitorium,

6703 HA Wageningen, The Netherlands

e-mail: Dane.Bicanic@wur.nl

D. Dimitrovski

Faculty of Technology and Metallurgy,

Ss. Cyril and Methodius University,

Rugjer Bošković 16,

1000 Skopje, Macedonia

\section{S. Luterotti}

Faculty of Pharmacy and Biochemistry, University of Zagreb,

Ante Kovačića 1,

10000 Zagreb, Croatia

\section{K. Marković}

Faculty of Food Technology and Biotechnology, Department of Food Quality Control and Nutrition, University of Zagreb, Pierottijeva 6,

10000 Zagreb, Croatia

\section{Introduction}

Lycopene, the unsaturated hydrocarbon with 11 conjugated and two non-conjugated double bonds, is one among approximately 600 carotenoids found in plants, bacteria, and algae. The results of various studies suggest that this compound plays a role in the prevention of different health issues (examples are chronic diseases, cardiovascular disorders, some cancers, etc.) in humans. ${ }^{1}$ As a potent antioxidant lycopene is presently marketed as a fortified nutritional supplement. ${ }^{2}$ Since tomatoes and the products derived from the thermally processed tomatoes are particularly rich in lycopene, the breeding of new tomato varieties with elevated levels of lycopene has become a world-wide trend.

\section{C. van Twisk \\ Product Quality Development, Agrotechnology and Food Sciences, Wageningen University, \\ Bomenweg 2-Biotechnion, \\ 6703 HA Wageningen, The Netherlands}

\author{
J. G. Buijnsters \\ Institute for Molecules and Materials (IMM), \\ Radboud University Nijmegen, \\ Heijendaalseweg 135, \\ 6525 AJ Nijmegen, The Netherlands
}

\section{O. Dóka}

Department of Physics and Mathematics,

University of West Hungary,

Deak F. sq.1,

9200 Mosonmagyaróvár, Hungary 
The importance of this phytonutrient requires the need for its quantification. The high-performance liquid chromatography (HPLC) is currently the analytical method most frequently used in practice. The HPLC offers superior specificity and sensitivity but it is elaborate and costly. This is because the extraction of lycopene from the product matrix is an imperative before the sample can be presented for the analysis. ${ }^{3,4}$ The isolation of lycopene is also a prerequisite for the spectrophotometric analysis. ${ }^{5}$ Recently, the combination of the VIS-NIR reflectance spectroscopy and the chemometrics was successfully used to quantify (in a non-destructive fashion) the lycopene content of fresh tomatoes. ${ }^{6}$ Likewise, the combined use of attenuated total reflectance spectroscopy in the infrared (at the $\mathrm{CH}$ out-ofplane deformation band of trans-lycopene close to $970 \mathrm{~cm}^{-1}$ ) and the multivariate techniques enabled rapid quantification of lycopene in fresh raw tomatoes. ${ }^{7}$ The FTRaman and NIR spectroscopies were also used to quantify lycopene in the tomato fruit. ${ }^{8}$ In addition, the surface distribution of carotenes in ripening tomatoes was retrieved by means of the non-invasive imaging spectrometry. ${ }^{9}$ Since most of lycopene in the tomato is found in the skin of the fruit, some investigators have used tristimulus colorimetry in an attempt to establish the correlation between the lycopene content and the chromaticity indices $\left(a^{*}, b^{*}\right.$, and $L^{*}$; or their transformations hue $h^{*}$ and chroma $\left.C^{*}\right) .{ }^{10,11}$ Good correlation between the transformed chromaticity indices $a^{* 4}$ and $\left(a^{*} / b^{*}\right)^{2.5}$ and the lycopene content of a pureed tomato was reported; the corresponding coefficients of determination were $R^{2}=0.945$ and $R^{2}=0.736$, respectively. ${ }^{12}$ For a ten-fold diluted tomato juice, linear correlation $\left(R^{2}=0.945\right)$ between the $a^{*} / b^{*}$ ratio and the trans-lycopene content (determined by spectrophotometric measurement of hexane extract) was observed. ${ }^{11}$ Finally, measured colorimetric index $a^{*}$ was used to predict the concentrations of lycopene in fresh tomatoes. ${ }^{6}$

In the recent years, the methodologies based on the photoacoustic (PA) and photothermal (PT) phenomena are gradually emerging as the non-conventional techniques for analysis of foods. ${ }^{13,14}$ Our research group centered at Wageningen University focuses on a development and putting in practice of novel PA- and PT-based techniques and instrumentation. The emphasis is mainly on the quantification of a specific analyte in foods without a need for using the extraction step.

The concepts behind the photoacoustic spectroscopy (PAS) and the optothermal window (OW) are essentially different from those encountered in the conventional spectroscopies. To a substantial number of researchers involved in the analysis of foods, the PAS and OW are still relatively unknown. Common to both above-stated methods is the fact that it is a fraction of energy absorbed by the sample from a periodically modulated source of incident radiation, that is converted into heat and eventually detected. When exposed to a periodically modulated radiation of selective wavelength, the condensed phased sample heats up/cools off periodically, which affects the pressure of a gas (the PA effect) in a hermetically sealed chamber (called the PA cell) above the sample. ${ }^{15}$ As far as the OW concept is being concerned, the heat generated in a sample causes the radial expansion of a piezoelectric disk that supports the very same sample. For PAS and OW detection schemes it holds true that the amplitude of the pressure fluctuation (called the PA signal) or the extent of the radial expansion (called the OW signal) correlates positively with the amount of heat generated in the sample. On its turn this heat depends in a first place on the concentration of the absorbing species in the sample, as well as on the incident power of the excitation source. It follows that the magnitude of the PA and OW signals is directly proportional to the concentration of a target analyte. Therefore, if the PA (or OW) signals have been measured for a number of samples and the concentration of specific target analyte in same samples is independently determined by an absolute method (such as for example the HPLC), one can construct the calibration curve. Such calibration curve enables the experimentalist to simply deduce the concentration of a same analyte in the unknown sample (without the need for the extraction step) from the experimentally obtained PA (or OW) signals. Because the information about the sample's absorbance in PA and OW studies is not obtained via the measurement of transmitted radiation (as it is normally done in a regular spectroscopy), the nontransparent samples can be readily studied simply as they are. Besides homogenization, no additional sample preparation procedure is needed before the analysis.

Since the pasty-like foods and liquids are among condensed phase samples suitable for PA and OW analysis, it is not surprising that different products derived from thermally processed tomatoes have received considerable attention by the investigators. The first application of the argon laser-based OW method for detection (at $502 \mathrm{~nm}$ ) of lycopene in the concentrated tomato puree was reported in 2003. ${ }^{16}$ The same detection concept was later extended to include products ranging from tomato juices and ketchups to triple tomato concentrates; the performance of the newly proposed method was compared to that of HPLC. ${ }^{17}$ The intrinsic precision achieved by the OW method was better than $0.2 \%$ and the repeatability comparable to that of HPLC (least overall error $0.86 \%$ ). Quite recently, the suitability of a compact OW setup based on the application of an inexpensive light-emitting diode as the source for excitation instead of argon ion laser was demonstrated. The approach proved capable of accurately assessing lycopene in the thermally processed tomato products with a lycopene 
content varying between 7 and $100 \mathrm{mg}$ per $100 \mathrm{~g}$ fresh product weight. ${ }^{18}$

Research study described in this paper focuses on exploring the potential of the PA and OW methods for the rapid detection of even lower concentration of lycopene. The two methods together with the micro-Raman spectroscopy and tristimulus colorimetry were used for a direct quantification of trans-lycopene in homogenates prepared from the raw tomatoes. Based on the obtained experimental data, an attempt was made to establish the correlation between the OW, PA and Raman signals, the chromaticity indices, and the absolute concentration of trans-lycopene content of same homogenates determined by the HPLC. The performance of different methods was statistically evaluated and compared.

\section{Materials and Methods}

\section{Sample Preparation}

Eight different sorts of fresh ripe tomatoes (different cultivars) investigated in this study were all grown in heated glass greenhouses. They were purchased either from the local grocery stores and public markets or obtained from the greenhouse growers; more details are disclosed in Table 1 . The average weight of cherry tomatoes was less than $25 \mathrm{~g}$ while cocktail tomato weighted about $50 \mathrm{~g}$ each. Several tomatoes from each of the eight sorts were used to prepare the homogenates that were assigned number codes ranging from 1 to 8 . Initially, tomatoes were washed and cut into the small cubes (volume of each about $1 \mathrm{~cm}^{3}$ ); the skin was not removed from the tomato. Then, mashed tomatoes were transferred into a cup and homogenized (at low speed for $2 \mathrm{~min}$ ) with Waring laboratory blender. The seeds were separated (filtration on a plastic sieve) from a pomace, and the 500 mbar external pressure was applied to remove oxygen bubbles, thereby improving the uniformity of homogenates. Tomato homogenates prepared in such a manner had a semiliquid consistency. Finally, all available quantity of every homogenate was distributed into smaller portions for analysis by each of the selected techniques: OW, colorimetry, PA spectroscopy, microRaman spectroscopy, and the reverse-phase HPLC. The first four methods are direct in the sense that, with the exception of homogenization step, no other sample handling procedure is required. The homogenates were kept at $4{ }^{\circ} \mathrm{C}$ in the refrigerator until the actual use. Because lycopene is susceptible to degradation (for example isomerization and oxidation), all measurements, with the exception of Raman analysis, were completed within a 2 days period.

\section{HPLC Analysis}

The reverse-phase HPLC system included the on-line degassing system (Alltech), a gradient pump (Spectra Physics P2000), YMC C30 column $(250 \times 4.6 \mathrm{~mm}$, S-5), auto sampler (Spectra Physics AS3000), and the photo diode array detector (UV6000LP). The wavelength of $476 \mathrm{~nm}$ was selected for detection purposes; measured data were processed by a computer using Chromeleon software. The gradient mobile phase (flow rate $1 \mathrm{ml} \mathrm{min}^{-1}$ ) consisted of eluents A (methanol/methyl tert-butyl ether (MTBE)-90/ 10 ) and $B$ (methanol/methyl tert-butyl ether-10/90). Antioxidant butylated hydroxytoluene (BHT) was added $(0.1 \%$ $w / v)$ to both eluents. The gradient sequence used in this experiment is shown in Table 2. Except BHT (from Sigma), other reagents such as methanol (from Biosolve BV) and MTBE (from Merck KGaA) were both of HPLC grade. The eluents were filtered through a $0.45-\mu \mathrm{m}$ membrane filter prior to the analysis. The mass of homogenate used for HPLC analysis (performed in triplicate) was approximately $1.25 \mathrm{~g}$. The extraction procedure performed under the subdued light conditions was initiated by adding $10 \mathrm{ml}$ hexane to the homogenate. After $10 \mathrm{~min}$ vortexing and $4 \mathrm{~min}$ centrifuging at 2,800 rpm, the supernatant was removed. This step was repeated (usually three times) until tomato flesh that has remained after centrifugation appeared practically colorless. The last step of the extraction sequence implies the addition of tetrahydrofuran (THF), which is miscible with water but also efficient in extracting carotenes. After the
Table 1 Different tomatoes used to prepare eight homogenates

\begin{tabular}{llll}
\hline Homogenate & Type & Flesh color & Consistency \\
\hline 1 & Crispy fresh & Orange & Firm \\
2 & Cherry round loose & Orange-red & Medium firm \\
3 & B-tomato & Light red & Firm \\
4 & Roma tomato & Red & Firm \\
5 & Farm tomatoes & Deep red & Firm \\
6 & Tomatoes from the market & Deep red & Firm \\
7 & Cherry truss & Deep red & Firm \\
8 & Cocktail tomato with high lycopene content & Deep red & Firm \\
\hline
\end{tabular}


Table 2 The gradient sequence used at a flow rate of $1 \mathrm{ml} \mathrm{min}-1$

\begin{tabular}{ll} 
Time (min) & Eluent A (\%) \\
\hline 0 & 88 \\
2 & 73 \\
4 & 57 \\
6 & 43 \\
8 & 20 \\
9.5 & 0 \\
14 & 0 \\
15 & 88 \\
25 & 88
\end{tabular}

treatment with THF the remaining tomato flesh was thick and gummy (the flesh was dehydrated from THF), making the extraction of remaining carotenoids difficult. Therefore, continuous addition of solvent to a homogenate has not resulted in a significant extraction of carotenoids. It is necessary to make sure that the extraction of carotenoids (with hexane) performed during all previous steps has been effective enough to allow addition of THF. Water confined within collected supernatant was eliminated by freezing and by removing the upper (colored) phase (hexane, THF, and extracted carotenes). The extract was vaporized using vacuum evaporator set to $40^{\circ} \mathrm{C}$ and 270 mbar. Finally, extracted carotenes were solubilized in a mobile phase $(88 \%$ eluent $\mathrm{A}$ and $12 \%$ eluent $\mathrm{B})$, filtered $(0.45 \mu \mathrm{m}$ diameter $)$, poured into amber vials, and directly analyzed.

The external standards of trans-lycopene and $\beta$-carotene were obtained from Sigma Chemicals. Due to dissolving problems of carotenoids it was necessary to optimize the preparation of calibrating solutions. Stock solution of translycopene in THF $\left(0.2 \mathrm{mg} \mathrm{ml}^{-1}\right)$ and that of $\beta$-carotene in THF (1.0 mg ml $\mathrm{m}^{-1}$ ), stored in liquid nitrogen, were first allowed to reach a room temperature. Then, an aliquot of solution was evaporated under nitrogen and diluted with an appropriate volume of hexane to obtain the absorbance readings of approximately $0.5 \mathrm{AU}$. The precise value of absorbance was determined by means of the UV-visible spectrophotometer (Cary 50 Bio from Varian) and the concentrations $\left(\mu \mathrm{g} \mathrm{ml}^{-1}\right)$ of all-trans lycopene and of beta carotene computed using the extinction coefficient $E_{1 \mathrm{~cm}}^{1 \%}=$ 3,450 at analytical wavelength of $476 \mathrm{~nm}$ (for lycopene) and $E_{1 \mathrm{~cm}}^{1 \%}=2,592$ at $453 \mathrm{~nm}$ (for $\beta$-carotene) respectively.

A series of standard solutions of trans-lycopene and of $\beta$-carotene were prepared starting with a highest concentration $\left(5.48 \mu \mathrm{g} \mathrm{ml}^{-1}\right.$ for trans-lycopene and $2.28 \mu \mathrm{g} \mathrm{ml}^{-1}$ for $\beta$-carotene) that was sequentially diluted (factor 2 ). In this manner, the concentration of trans-lycopene and of $\beta$-carotene in standard solutions varied between 5.48 and $0.09 \mu \mathrm{g} \mathrm{ml}^{-1}$ and between 2.28 and $0.07 \mu \mathrm{g} \mathrm{ml} \mathrm{m}^{-1}$, respectively. The "purity degree" of standard solutions was expressed in terms of the peak area of the target carotenoid (trans-lycopene or $\beta$-carotene) relative to the total area of chromatogram. The peak areas of calibration solutions containing the highest and the lowest concentrations of trans-lycopene and of $\beta$-carotene are both within a range of values obtained from the homogenates studied here. The quantifications of all-trans lycopene and of $\beta$-carotene were accomplished from the experimentally determined area of chromatographic peaks and the calibration curves.

\section{Experimental Setup for OW and PA Measurements}

The experimental setup (Figure 1) used for PA and OW measurements consisted of (1) a free running Lexel 85 argon laser (long-term power stability better than $0.5 \%$ ), (2) a set of plane mirrors used to guide/direct the laser beam, (3) the mechanical chopper (Stanford Research) for the periodical modulation of the laser beam, and (4) the detector (either OW or PA cell) with the processing electronics. The laser beam (about $2 \mathrm{~mm}$ in diameter) was not focused. The 502-nm emission line of this laser was chosen for selective excitation. At this wavelength the lycopene is strongly absorbing while at the same time the influence of the interfering pigment (beta carotene) is minimized. For purposes of the OW measurement, the homogenate was simply deposited on a surface of the sapphire disk. The response of $\mathrm{OW}$ and PA cell was measured at desired modulation frequency using the EG\&G 5110 dual phase lock-in amplifier (integration time $1 \mathrm{~s}$ ) and the OW (or PA) signal processed by the computer.

The heart of the experimental setup is the non-resonant PA cell. Its major components are the sample tray (stainless steel plate $8 \mathrm{~mm}$ thick and $60 \mathrm{~mm}$ in diameter) provided with a central semispherical cavity ( $6 \mathrm{~mm}$ diameter), the air compartment (volume about $100 \mathrm{~mm}^{3}$ ) above the tray, quartz entrance window (2 mm thick and $25.4 \mathrm{~mm}$ in

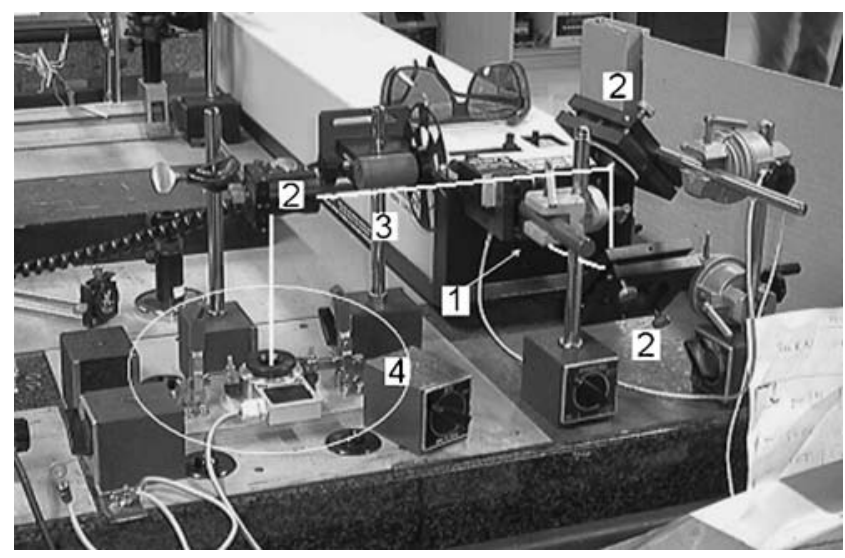

Fig. 1 The experimental setup used in PA studies of tomato homogenates: c.w. argon laser (1), three plane mirrors (2), mechanical chopper (3), and the PA cell (4) 
diameter) highly transparent to 502-nm incident laser radiation, and a sensitive Sennheiser KE 211-4 electret microphone. The air chamber and a microphone are connected via the 20 -mm-long cylindrical channel $2 \mathrm{~mm}$ in diameter. The PA cell was sealed using an "O" ring and the two clamps; such a construction removed the possibility for pressure leakage, ensuring thereby that all heat generated by the absorption indeed contributed to a pressure buildup in the air compartment above the sample. The modulation frequency used in the PA and OW experiments was 25 and $9 \mathrm{~Hz}$, respectively. At $25 \mathrm{~Hz}$ thermal diffusion length $\mu_{\text {air }}$ in the air, defined as $\mu_{\text {air }}=\left(\alpha_{\text {air }} / \pi f\right)^{1 / 2}$ is about $350 \mu \mathrm{m}$ at the room temperature and the atmospheric pressure. This is shorter than a $3.0-\mathrm{mm}$ distance between the surface of the sample and the rear side of the quartz window. By assuming $\alpha_{\mathrm{hom}}=$ $1.44 \times 10^{-7} \mathrm{~m}^{2} \mathrm{~s}^{-1}$ for thermal diffusivity of tomato homogenate (value is close to that of water) at the room temperature, one obtains $\mu_{\text {hom }}=42 \mu \mathrm{m}$ at $25 \mathrm{~Hz}$. This value is shorter than both the physical thickness $L(6 \mathrm{~mm})$ of tomato homogenate and the optical penetration depth $1 / \beta=$ $540 \mu \mathrm{m}$ (with $\beta=18.5 \mathrm{~cm}^{-1}$ at $502 \mathrm{~nm}$ as the absorption coefficient of homogenate per unit length). This value for $\beta$ has been calculated by taking $2 \times 10^{5} 1 \mathrm{~mol}^{-1} \mathrm{~cm}^{-1}$ for the extinction coefficient of lycopene (its molecular weight is 536.87) and $5 \mathrm{mg}$ per $100 \mathrm{~g}$ as a typical content of lycopene in fresh tomato homogenate. The actual laser power incident on tomato homogenate was $17 \mathrm{~mW}$ as measured by a Spectra Physics 407A power meter. Both experimental setups used for OW and PA measurements were optimized by maximizing the optical alignment.

\section{Measurement Protocol Used in OW and PA Studies}

The PA and OW measurements were conducted according to a well-defined protocol schematically shown in Figure 2. With the tomato homogenate loaded (a small quantity of homogenate is adequate for analysis), the beam of the laser was blocked for $10 \mathrm{~s}$ to allow the lock-in signal to reach a zero signal level. For the next $10 \mathrm{~s}$ beam is unblocked, and the amplitude of the non-zero lock-in signal reaches its end

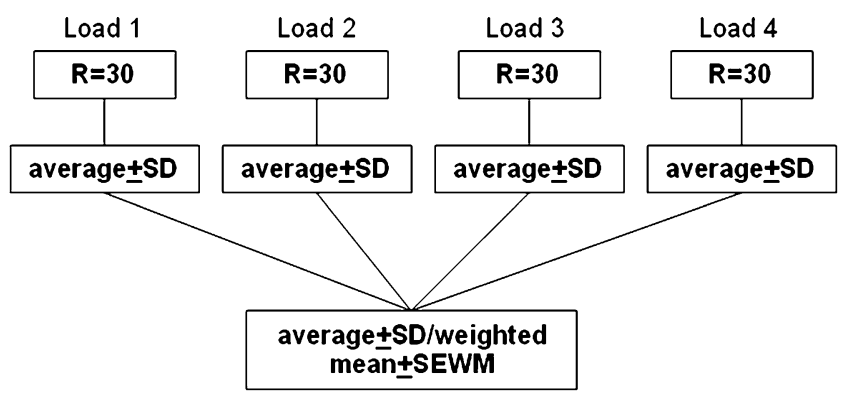

Fig. 2 Schematic diagram of the measurement protocol used in OW and PA studies of tomato homogenates. $R$ number of measurements value. During the following $30 \mathrm{~s}, 30(R=30)$ successive readings of the lock-in signal were taken, and the corresponding average and standard deviation (SD) were calculated. Standard deviation obtained in such manner is indicative for the instrumental precision achievable by such "single-load" measurement. After completing the first 30 measurements, homogenate was removed, and a surface of OW sensor (or the sample tray of the PA cell) was cleaned. Fresh quantity of the same homogenate was then deposited, and the entire procedure described above was repeated. Four such replicate PA and OW measurements were carried out with each of eight homogenates. Data collected were used to compute the average signal and SD as well as the standard error of the weighted mean (SEWM). Both SD and SEWM are indicative for the repeatability, i.e., precision achievable in "multiple loads" measurements.

It is customary to normalize measured PA (or OW) signals (abbreviated $S_{\text {sample }}$ ) by rationing them to a signal $S_{\text {ink }}$ obtained under identical experimental conditions from a black drawing ink that serves as a strongly absorbing reference. From the normalized signal $S=S_{\text {sample }} / S_{\text {ink }}$ one can compute the dimensionless product $\beta \mu$ for a given homogenate according to ${ }^{19,20}$ :

$$
\beta \mu=\frac{2}{\sqrt{\left(2 / S^{2}\right)-1}-1}
$$

After computing $\beta \mu$ for all the homogenates, the experimentalist can construct the plot displaying $\beta \mu$ product as a function of lycopene concentration (determined independently by the HPLC) in the very same homogenates. In fact such a plot represents the calibration curve that can be readily used to rapidly deduce the concentration of lycopene in an arbitrary tomato homogenate from measured PA or OW data. It is essential that the experimental conditions (sample-laser beam geometry, laser wavelength and power, the modulation frequency, etc.) during measurements are the same as those used for the calibration procedure.

\section{Colorimetric Analysis}

Automated measurements (ColorFlex Utility software) of tomato's chromaticity indices $\left(L^{*}, a^{*}\right.$, and $\left.b^{*}\right)$ and of transformed indices (hue angle $h^{*}$ and chroma $C^{*}$ ) were conducted on a ColorFlex-CFLX-45 colorimeter $\left(45^{\circ} / 0^{\circ}\right)$ manufactured by Hunter Associates Laboratory Inc. The instrument was configured for " $\mathrm{C}$ " standard illuminant with the $2^{\circ}$ observer. The basic thought behind colorimetric measurements is the assumption that the red color is related to the tomato's lycopene content. White and black standard tiles were used to calibrate the instrument before each measurement. 
Table 3 The concentration (measured by the HPLC and expressed in $\mu \mathrm{g}$ per $\mathrm{g}$ of fresh weight (FW) homogenate) of trans-lycopene and beta carotene in tomato homogenates; the corresponding relative standard deviations (RSD) are also shown

\begin{tabular}{llllll}
\hline \multicolumn{1}{l}{ trans-lycopene } & & & $\beta$-carotene \\
\cline { 1 - 2 } \cline { 5 - 6 } Tomato homogenate & Content $\left(\mu \mathrm{g} \mathrm{g}^{-1} \mathrm{FW}\right)$ & $\mathrm{RSD}(\%)$ & & Content $\left(\mu \mathrm{g} \mathrm{g}^{-1} \mathrm{FW}\right)$ & RSD $(\%)$ \\
\hline 1 & 9.62 & 6.40 & & 1.49 & 4.63 \\
2 & 13.55 & 6.60 & & 1.01 & 2.11 \\
3 & 19.98 & 1.69 & & 1.46 & 2.75 \\
4 & 32.90 & 0.53 & & 1.87 & 2.47 \\
5 & 43.45 & 0.54 & & 2.46 & 3.25 \\
6 & 56.99 & 0.81 & & 3.95 & 0.34 \\
7 & 56.11 & 1.00 & & 4.95 & 2.10 \\
8 & 54.53 & 2.54 & & 4.27 & 4.46 \\
\hline
\end{tabular}

Measurement Protocol for Colorimetric Studies

A glass cup filled with the tomato homogenate was placed above the light source and covered with a black cover that served as a light trap. The color of tomato homogenate was measured in the reflectance mode with the cup's bottom facing the incoming light. Twenty-five $(R=25)$ consecutive color readings were taken during the 4-min period (approximately one measurement per $10 \mathrm{~s}$ ) before calculating the corresponding average. The remaining procedure used (in triplicate measurements) to access the average and its standard deviation was the same as that described above for the PA and OW measurements (see also Figure 2).

\section{Micro-Raman Spectroscopy}

Tomato homogenates were placed into a home-made PVC holder (40 mm diameter) provided with the central semispherical cavity $4 \mathrm{~mm}$ in diameter. In a typical micro-Raman

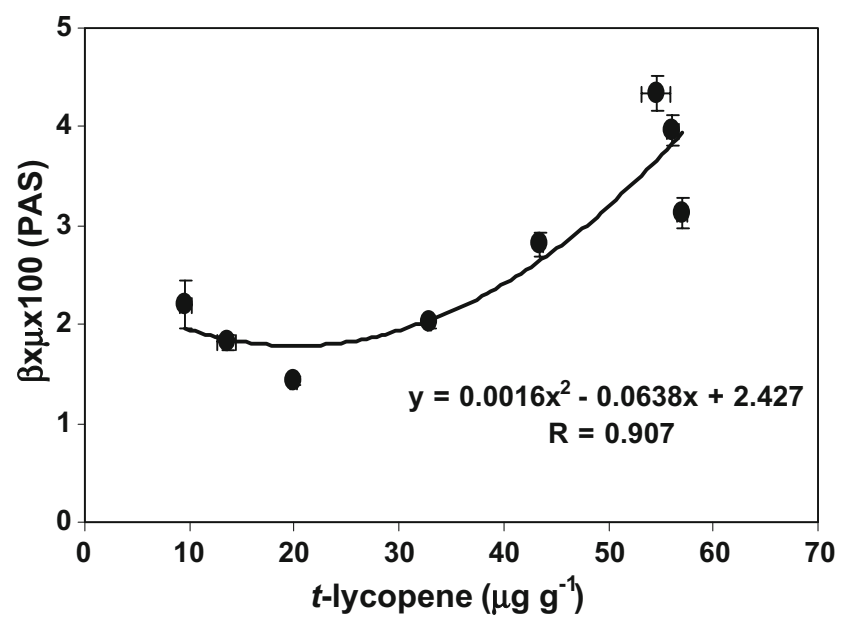

Fig. 3 Hundred-fold $\beta \mu$ product obtained from measured PA data plotted versus the absolute concentration of trans-lycopene (determined independently by the HPLC measurement) in the homogenates of raw tomatoes. Mean \pm SD data; PAS $n=4$, HPLC $n=3$ measurement, $1 \mathrm{~mW}$ of $514 \mathrm{~nm}$ laser radiation from an air cooled argon laser was focused to a spot $(50 \mu \mathrm{m}$ in diameter) on a homogenate itself; the exposure time was $10 \mathrm{~s}$. Raman backscattered light was collected and analyzed by a Renishaw 1000 micro-Raman spectroscope equipped with the integrated Leica DMLM imaging microscope and thermoelectrically cooled CCD array detector. Spectral data were recorded in the 950 to $1,750 \mathrm{~cm}^{-1}$ range with $1 \mathrm{~cm}^{-1}$ resolution. Raman response of lycopene is characterized by the three strong high-frequency Stokes-shifted signals originating from $\mathrm{C}=\mathrm{C}$ and $\mathrm{C}-\mathrm{C}$ stretches of molecule's polyene backbone and from a bending vibration of the methyl group. The corresponding positions of these Raman bands are expected close to 1,520, 1,155 , and $1,008 \mathrm{~cm}^{-1}$, respectively. ${ }^{8}$ The intensity of Raman peaks (expressed in terms of photon counts) was quantified after subtracting the photoluminescence background. Each homogenate was studied in triplicate, and the average and standard deviation were calculated.

\section{Results}

Lycopene and $\beta$-Carotene Content of Tomato Homogenates

Table 3 shows the content and the relative standard deviation of trans-lycopene and beta carotene of eight tomato homogenates as determined by the HPLC method. Data expressed in $\mu \mathrm{g}$ of compound per $1 \mathrm{~g}$ fresh weight (FW) of tomato homogenate represent the average of three independent HPLC measurements. The trans-lycopene (its concentration varies from 9.62 to $56.99 \mu \mathrm{g} \mathrm{g}^{-1} \mathrm{FW}$ ) is the most abundant carotenoid followed by the $\beta$-carotene, as expected.

Trans-Lycopene Content of Tomato Homogenates Measured by OW and PA

At a given incident laser power $(17 \mathrm{~mW}$ at $502 \mathrm{~nm})$ and $9 \mathrm{~Hz}$ modulation frequency, the OW signals obtained from eight tomato homogenates were typically between 50 and 
Table 4 The coefficients of correlation for fitted relationships between the chromaticity indices (and their transformations) and the trans-lycopene content (determined by the HPLC and extending from 9.62 to $56.99 \mu \mathrm{g} \mathrm{g}^{-1} \mathrm{FW}$ ) of eight tomato homogenates

\begin{tabular}{|c|c|c|c|c|c|}
\hline \multirow[t]{2}{*}{ Correlation } & \multicolumn{5}{|c|}{ Coefficients of correlation for lycopene fit } \\
\hline & Linear & Logarithmic & Polynomial & Power & Exponential \\
\hline$L^{*}$ & 0.1776 & 0.1810 & 0.1838 & 0.1810 & 0.1810 \\
\hline$a^{*}$ & 0.8233 & 0.8995 & 0.9710 & 0.9127 & 0.8184 \\
\hline$b^{*}$ & 0.4934 & 0.5808 & 0.6582 & 0.5859 & 0.4969 \\
\hline$C^{*}$ & 0.8853 & 0.9506 & 0.9720 & 0.9556 & 0.8700 \\
\hline$h^{*}$ & 0.6835 & 0.7444 & 0.7683 & 0.7713 & 0.7131 \\
\hline Reflectance at $500 \mathrm{~nm}$ & 0.5681 & 0.6231 & 0.6493 & 0.5795 & 0.5434 \\
\hline$a^{*^{2}}$ & 0.8097 & 0.8681 & 0.9540 & 0.9127 & 0.8184 \\
\hline$a^{*^{4}}$ & 0.7370 & 0.7675 & 0.8057 & 0.9127 & 0.8184 \\
\hline$\left(a^{*}\right)^{1 / 2}$ & 0.8231 & 0.9084 & 0.9770 & 0.9127 & 0.8184 \\
\hline$a^{*} / b^{*}$ & 0.7260 & 0.7821 & 0.8012 & 0.7465 & 0.6864 \\
\hline$\left(a^{*} / b^{*}\right)^{2}$ & 0.7547 & 0.8042 & 0.8191 & 0.7465 & 0.6864 \\
\hline$\left(a^{*} / b^{*}\right)^{2.5}$ & 0.7647 & 0.8100 & 0.8234 & 0.7465 & 0.6864 \\
\hline$\left(a^{*} / b^{*}\right)^{6.5}$ & 0.7557 & 0.7639 & 0.7786 & 0.7465 & 0.6864 \\
\hline $1000 * a^{*} /\left(b^{*}+L^{*}\right)$ & 0.8213 & 0.8863 & 0.9130 & 0.8651 & 0.7875 \\
\hline$C^{* 2}$ & 0.8213 & 0.9110 & 0.9560 & 0.9127 & 0.8184 \\
\hline
\end{tabular}

$90 \mu \mathrm{V}$. The homogenates rich in lycopene produced high OW signals (about 80 to $90 \mu \mathrm{V}$ ) and vice versa. In the case of PA measurements $(17 \mathrm{~mW}$ at $502 \mathrm{~nm})$ conducted at $25 \mathrm{~Hz}$, PA the signals range between 20 and $80 \mu \mathrm{V}$. Under such experimental conditions no evidence has been found for degradation of lycopene in the course of OW or PA measurements. The dependence of the $\beta \mu$ product obtained in PA measurements on a content of trans-lycopene in the eight tomato homogenates is shown in Figure 3. A similar curve was obtained when using data from the $\mathrm{OW}$ measurements. The two sets of data were fitted by a second-order polynomial yielding $R^{2}=0.82$ as a coefficient of determination of both, PA spectroscopy and OW. Since the absorption of lycopene at $502 \mathrm{~nm}$ is significantly higher than that of beta carotene, one can state that OW and PA signals are largely determined by trans-lycopene in homo-

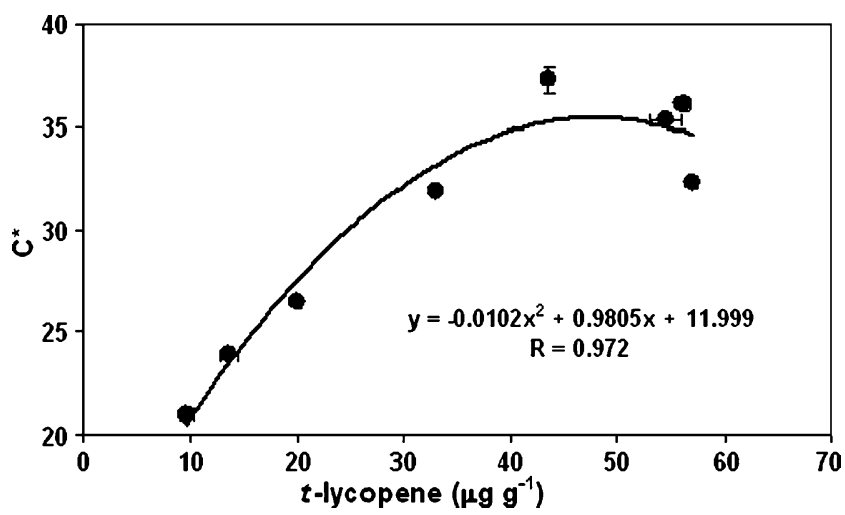

Fig. 4 The functional dependence between transformed colorimetric parameter $C^{*}$ (chroma) and the absolute concentration of translycopene (determined independently by HPLC measurement) in raw tomato homogenates. Mean $\pm \mathrm{SD}$ data; colorimetry $n=3$, HPLC $n=3$ genates. In homogenates with trans-lycopene content exceeding $20 \mu \mathrm{g} \mathrm{g}^{-1} \mathrm{FW}$, the magnitude of OW and PA signals was directly proportional to the total absorbance.

Colorimetric Assessment of Trans-Lycopene in Tomato Homogenates

Table 4 displays the extent of correlation between the chromaticity indices $a^{*}, b^{*}, L^{*}, C^{*}, h^{*}$ (as well as different combinations of these quantities), and the trans-lycopene content of eight tomato homogenates. Measured data were fitted with the linear, polynomial, logarithmic, power, and exponential functions. High correlations were obtained when fitting the experimental data (Figures 4 and 5) for chroma $C^{*}$ and $\left(a^{*}\right)^{1 / 2}$ by a second-order polynomial. The tendency toward saturation was observed for the lycopene-

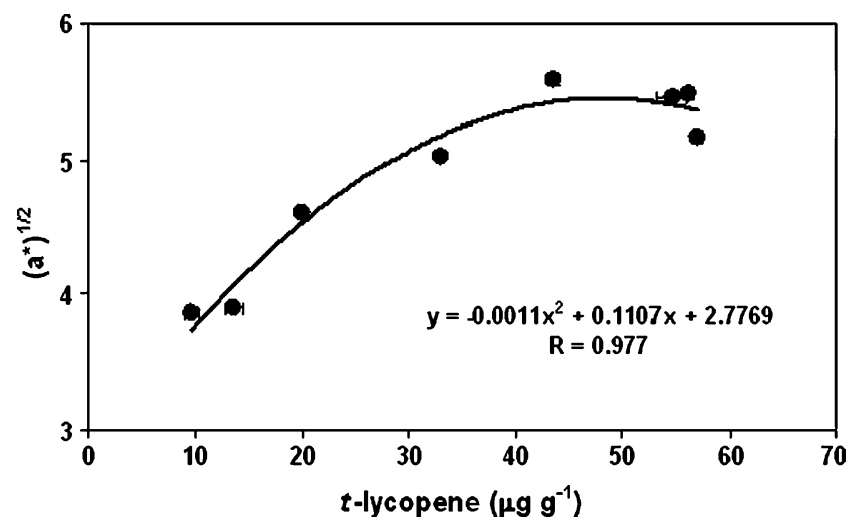

Fig. 5 The colorimetric index $\left(a^{*}\right)^{1 / 2}$ plotted versus the absolute concentration of trans-lycopene (determined independently by HPLC measurement) in raw tomato homogenates. Mean \pm SD data; colorimetry $n=3$, HPLC $n=3$ 


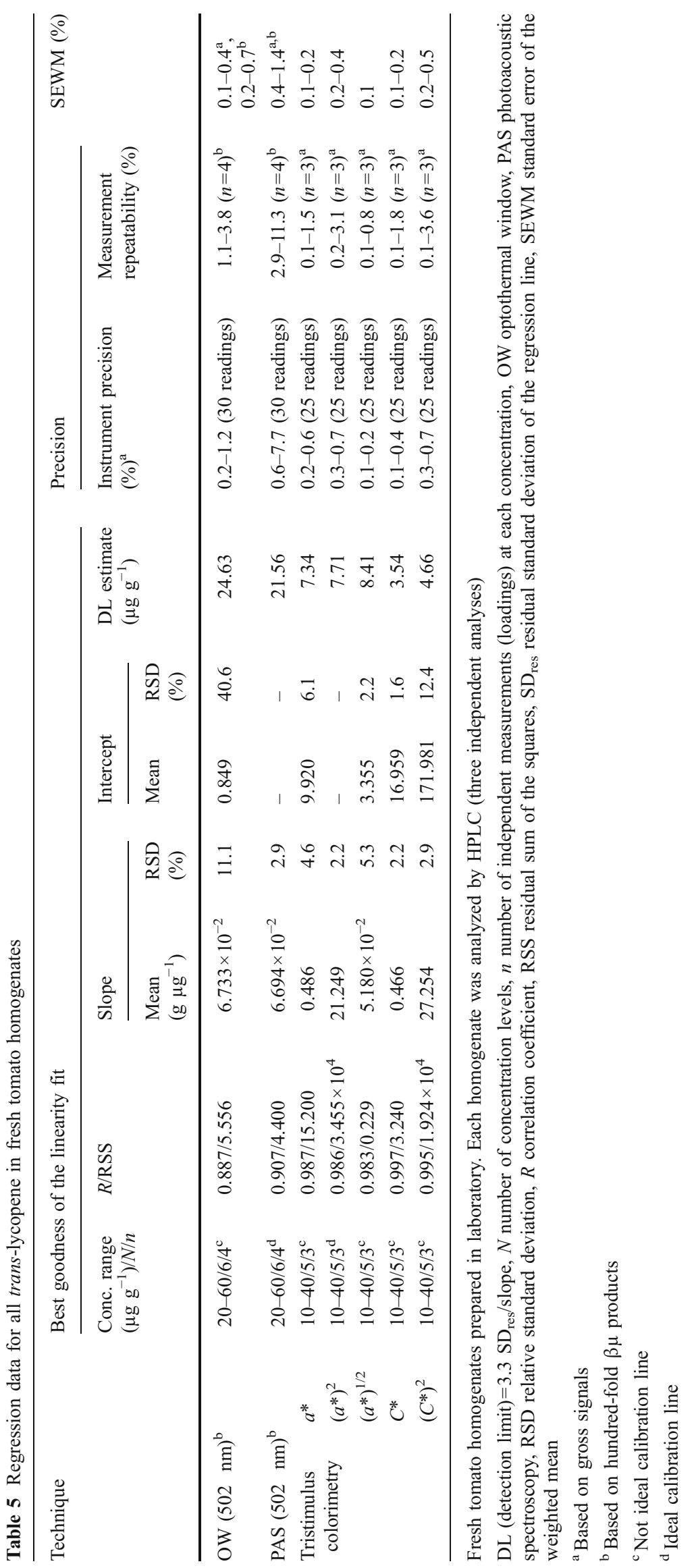


rich homogenates. However, the linear correlation better fits the data obtained from homogenates with the translycopene concentration not exceeding $40 \mu \mathrm{g} \mathrm{g}^{-1} \mathrm{FW}$. The instrumental precision and the analysis repeatability of colorimetry were evaluated by calculating relative standard deviations (RSDs; \%) as shown in Table 5.

\section{Raman Results}

The most intense characteristic Raman band was that appearing at $1,520 \mathrm{~cm}^{-1}$ associated with the $\mathrm{C}=\mathrm{C}$ stretching vibration $\left(v_{1}\right)$ of lycopene. Plotting the intensity of the peak (after correction for the contribution of the background) versus the concentration of trans-lycopene in homogenate points to a poor correlation $\left(R^{2}=0.52\right)$. This is most likely due to a fact that because of unforeseen circumstances, Raman measurements could not be performed until 1 week after completing the PA, OW, and colorimetric measurements; during all that time the homogenates were kept at $-22^{\circ} \mathrm{C}$. Other factors that might be responsible for a poor correlation include the small spot size of the focused laser radiation (in comparison with the scale of sample's own uniformity), high power density of the laser, and too long exposure.

\section{Conclusion}

The extent of correlation between the four selected direct methods and a widely used HPLC for quantifying translycopene in tomato homogenates was explored extensively. The fraction of trans-lycopene in homogenates studied here varied between $87 \%$ and $95 \%$; the remaining part is trans beta carotene. The sum of the trans-lycopene and trans- $\beta$ carotene approximates the total carotenoid (TC) content. Detailed analysis of analytical figures of merit with regard to the concentration of trans-lycopene (dominant carotenoid in tomato homogenates) is presented in Table 5; the performances of various methods are comparable.

From the data shown in Tables 4 and 5, the following conclusions can be drawn. As far as the complete set of data for trans-lycopene (concentration range extending from 10 to $60 \mu \mathrm{g} \mathrm{g}^{-1} \mathrm{FW}$ ) is concerned, the results suggest that the best fit is obtained with polynomial functions. The linear regression analysis (with a moderate coefficient of correlation) is only achievable for a reduced concentration range (Table 5). This is accompanied by a modest RSD for PAS and the relatively high detection limit (DL) values for both OW and PA methods. The outcome of this study provides the first experimental evidence that, the OW and PA methods the usability of which for determination of lycopene in thermally processed tomato products has already been proven, might possibly also be used for a routine screening of tomato homogenates with the translycopene concentration between 20 and $60 \mu \mathrm{g} \mathrm{g}^{-1}$. These two methods are, as predicted previously, feasible for a routine screening of tomato homogenates with the translycopene concentration between 20 and $60 \mu \mathrm{g} \mathrm{g}^{-1} \mathrm{FW} .^{17}$ It is anticipated that the overall performance of the OW and PA methods can be further improved. For example, additional enhancement in the sensitivity of OW measurements is achievable if the currently used sapphire disk is replaced by another material with larger radial expansion coefficient. As to the PA detector, the gain in sensitivity is expected after (1) reducing the volume of a gas compartment above the homogenate, (2) utilization of a more sensitive microphone, and (3) using higher incident laser power. Research of this matter is in progress.

Markedly better linear correlations were observed for chromaticity index $a^{*}$ and transformed index $C^{*}$ and $\left(C^{*}\right)^{2}$. A good linear correlation was observed for tomato homogenates with the trans-lycopene concentration between 10 and $40 \mu \mathrm{g} \mathrm{g}^{-1} \mathrm{FW}$. Unlike in a case of $\mathrm{OW}$ and PA spectroscopy, the quantities such as chroma $C^{*}$ and $\left(C^{*}\right)^{2}$ exhibit good precision, and favorable DL values (3.5-4.7 $\mu \mathrm{g} \mathrm{g}^{-1}$ ). Both $a^{*}$ and $C^{*}$ as well as some of transformed quantities have comparable calibration sensitivity, instrumental precision $(0.1-0.7 \%)$ and measurement repeatability. The latter is considerably better for chromaticity indices than for OW and PA methods. The repeatability imprecision for $\left(a^{*}\right)^{2}$ and $\left(C^{*}\right)^{2}$ did not exceed $3.6 \%$; furthermore the RSD values for $a^{*}$ and $C^{*}$ were between $0.1 \%$ and $1.8 \%$. The SEWM values for indices are good and span the range from $0.1 \%$ to $0.5 \%$.

The favorable instrumental precision $(0.1-0.4 \%)$ achieved in colorimetric measurement of $C^{*}$, high measurement repeatability $(0.1-1.8 \%)$, and favorable DL $\left(3.54 \mu \mathrm{g} \mathrm{g}^{-1}\right.$ trans-lycopene) confirm the potential of chroma $C^{*}$ for assaying trans-lycopene (or TC). In terms of the RSS, the slope, the intercept precision, detection limit DL, and SEWM, chroma $C^{*}$ offers more favorable values than $\left(C^{*}\right)^{2}$. It is for this reason that chroma $C^{*}$ can be proposed as a tool for a reliable quantitation of trans-lycopene (total carotenoids) in the fresh tomato homogenates.

Acknowledgments The authors are grateful to Tom Bessems, Hans de Rooij, Hans Meijer, Reinoud van Hummelen, Hennie Boshoven, Edo Gerkema (all associated with Wageningen University), and to András Miklós (Steinbeis Transfer Centre Applied Acoustics, Stuttgart, Germany) for their perpetual, highly skilled technical assistance as well as for the frequent, most stimulating discussions on a variety of relevant issues.

Open Access This article is distributed under the terms of the Creative Commons Attribution Noncommercial License which permits any noncommercial use, distribution, and reproduction in any medium, provided the original author(s) and source are credited. 


\section{References}

1. V.R. Preedy, R.R. Watson, Tomato and Tomato ProductsNutritional, Medicinal and Therapeutic Properties (Science Publishers, Enfield, New Hampshire, 2008)

2. R. Levin, G. Cheshire, The Red Bodyguard: the Amazing Health Promoting Properties of the Tomato (Icon Books, Cambridge, 2008)

3. A.I. Olives Barba, M. Cámara Hurtado, M.C. Sánchez Mata, V. Fernández Ruiz, M.L.S. de Tejadab, Food Chem 95, 328-336 (2006)

4. C.H. Azevedo-Meleiro, D.B. Rodriguez-Amaya, J Food Comp Anal 17, 385-396 (2004)

5. G. Sadler, J. Davis, D. Dezman, J Food Sci 55, 1460-1461 (1990)

6. A. Clément, M. Dorais, M. Vernon, J Agric Food Chem 56, 15381544 (2008)

7. Y. Halim, S.J. Schwartz, D.M. Francis, N.A. Baldauf, L.E. Rodriguez-Saona, J AOAC Int 89, 1257-1264 (2006)

8. M. Baranska, W. Schütze, H. Schulz, Anal Chem 78, 8456-8461 (2006)

9. G. Polder, G.W.A.M. van der Heijden, H. van der Voet, I.T. Young, Postharvest Biol Technol 34, 117-129 (2004)
10. R. Arias, L. Tung-Ching, L. Logendra, H. Janes, J Agric Food Chem 48, 1697-1702 (2000)

11. D. M. Barrett, G. E. Anthon, in Color Quality of Fresh and Processed Foods, ed. by C.A. Culver, R.E. Wrolstad, ACS Symposium Series 983, 2008, pp. 131-139

12. J.R. Hyman, J. Gaus, M.R. Foolad, J Amer Soc Hort Sci 129, 717-723 (2004)

13. H.K. Michaelian, Photoacoustic Infrared Spectroscopy (WileyInterscience, Hoboken, 2003)

14. E. S. Bialkowski, in A Series of Monographs on Analytical Chemistry and Its Applications, ed. by J. D. Winefordner (WileyInterscience, Hoboken, 1996), p. 134

15. D.P. Almond, P.M. Patel, Photothermal Science and Techniques (Chapman \& Hall, London, 1996)

16. D. Bicanic, M. Anese, S. Luterotti, D. Dadarlat, J. Gibkes, M. Lubbers, Rev Sci Inst 74, 687-689 (2003)

17. D. Bicanic, V. Fogliano, S. Luterotti, J. Swarts, G. Piani, G. Graziani, J Sci Food Agric 85, 1149-1153 (2005)

18. D. Bicanic, R. Cuypers, S. Luterotti, M. Šporec, A. Zoppi, J Vugec, Acta Chim Slov 55, 468-473 (2008)

19. G.F. Kirkbright, M.J. Adams, Eur Spectr News 14, 22-27 (1977)

20. S.P. Belton, S.F. Tanner, Analyst 108, 591-596 (1983) 